\title{
Spotlight on brodalumab in the treatment of plaque psoriasis: the evidence to date
}

This article was published in the following Dove Press journal:

Clinical, Cosmetic and Investigational Dermatology

\author{
M Galluzzo* \\ S D'Adamio* \\ A Massaro \\ A Piccolo \\ L Bianchi \\ M Talamonti \\ Dermatology, Department of "Medicina \\ dei Sistemi", University of Rome Tor \\ Vergata, Rome, Italy \\ *These authors contributed equally to \\ this work
}

\section{Video abstract}

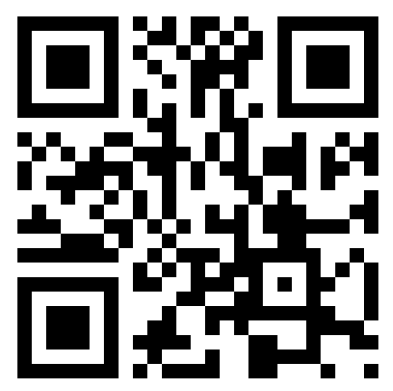

Point your SmartPhone at the code above. If you have a $Q R$ code reader the video abstract will appear. Or use: https://youtu.be/XdU4cyB54FY
Correspondence: M Galluzzo Dermatology, Department of "Medicina dei Sistemi", University of Rome Tor Vergata, Viale Oxford, 8I, Rome 00133, Italy

Tel +390620902743

Fax +390620902742

Email marco.galluzzo83@gmail.com

\begin{abstract}
The IL-17/IL-23 axis is now understood to influence psoriasis, and the development of novel IL-17 inhibitor medications marks a sea change in the treatment of psoriasis. Brodalumab is a recombinant, fully human immunoglobulin IgG2 monoclonal antibody specifically targeted against IL-17RA. This article discusses the mechanism of action and the efficacy and safety profile of brodalumab presented in the literature. Brodalumab, the latest approved anti-IL-17-class medication, is the only one that exerts its effects on IL-17C as well as on IL-17A and IL-17F, blocking the shared IL-17 receptor A. In this sense, considering the recent evidence, brodalumab could have beneficial effects not only on psoriasis, but also on atopic dermatitis. It could also serve as a therapeutic alternative in patients who develop paradoxical eczematous reactions or atopic-like dermatitis during treatment with other anti-IL-17A (secukinumab, ixekizumab).
\end{abstract}

Keywords: psoriasis, biologics, anti-IL17RA, brodalumab, PASI100, atopic dermatitis

\section{Background}

Psoriasis, a chronic inflammatory disease, affects $10 \%$ of the world population. Patients with psoriasis not only deal with pain and discomfort; they are also often socially stigmatized, psychologically distressed and isolated from the community. ${ }^{1}$ The presence of psychological distress, highlighting with depression and anxiety, is a wellknown aspect of psoriasis and has been reported extensively. ${ }^{2,3}$ Psoriasis is also related to an increase in comorbidities, including obesity, metabolic syndrome, cardiovascular disease, psoriatic arthritis (PsA) and autoimmune disease. About $20 \%$ of patients with psoriasis have been estimated to have moderate to severe disease. ${ }^{4,5}$ The most common form of psoriasis is the chronic plaque variant, involving $90 \%$ of psoriasis patients. The clinical appearance of the disease is dominated by raised, well-demarcated erythematous plaques with a variable amount of adherent silver scales. Plaques may have steep, "drop-off" edges or be relatively smooth, with a silvery white scale that is characteristic of the disease. The removal of scale may result in pinpoint bleeding known as the Auspitz sign. Lesions tend to be located on extensor surfaces, with the elbows and knees symmetrically involved in many cases, although it can present on all body surfaces. ${ }^{6-8}$ Psoriasis is a chronic disease, and long-term treatment is necessary.

Changes to the IL-23/Th17 axis appear to play a primary role in the pathogenesis of psoriasis. Along with TGF $\beta$ and the interleukin 6 (IL-6) cytokine, the upstream cytokine IL-23 is fundamental in developing and maintaining a pathogenic Th17 cell phenotype from naive CD4+ T cells. This subset of T-helper cells, which produces IL-17, is key in coordinating the inflammatory development of 
psoriasis. $^{9,10}$ There are six cytokines (IL-17A-F) and five receptors (IL-17RA-RE) in the IL-17 cytokine family. The overproduction of ILs (17A, 17F, and 17A/F) causes keratinocytes to proliferate, leading to the formation of psoriatic plaques. Blocking IL-17RA impedes the activity of several members of the IL-17 cytokine family, thereby normalizing inflammation of the skin. ${ }^{11}$

Biologic therapies target key mechanisms of a disease's pathogenesis. Psoriasis may be treated with biologics that disrupt tumor necrosis factor (TNF) (adalimumab, etanercept, infliximab, golimumab, certolizumab) or IL-17 (brodalumab, ixekizumab, and secukinumab), or those that inhibit IL-23p19 (guselkumab) or IL-12/23 (ustekinumab). ${ }^{12}$

\section{Methods}

The PubMed databases were searched for articles regarding brodalumab and the ClinicalTrials.gov database was searched for trials. Additional publications were collected from references identified in articles and related citations in PubMed. New data presented at the 2018 American Academy of Dermatology (AAD) and the European Academy of Dermatology and Venereology (EADV) meetings were also included.

\section{Mechanism of action and regulatory affairs}

Brodalumab is a recombinant, fully human immunoglobulin IgG2 monoclonal antibody specifically targeted against IL-17RA.

Brodalumab was approved in Japan on 4 July 2016 to treat psoriasis vulgaris, psoriatic arthritis, pustular psoriasis and psoriatic erythroderma. It was later approved by the FDA on 15 February 2017 for the treatment of moderate-to-severe plaque psoriasis. On 17 July 2017, the European Commission authorized marketing of brodalumab throughout the European Union for the treatment of moderate-to-severe plaque psoriasis. ${ }^{13}$

The recommended dose is a subcutaneous injection of $210 \mathrm{mg}$ administered once per week for three weeks, followed by $210 \mathrm{mg}$ every 2 weeks.

\section{Safety and efficacy data in Phase III studies and new insights of literature}

In this review, we analyze the most important Phase 3 studies regarding brodalumab and we include recent results isolated from these trials. AMAGINE-1, a Phase III, double-blind, randomized, placebo-controlled, safety/efficacy study (NCT01708590), consisted of a 12-week induction period followed by a withdrawal-retreatment period lasting up to 52 weeks. The patients ranged from 18 to 75 years old at screening and had stable moderate-to-severe plaque psoriasis for more than six months before the initial dose of brodalumab. The body surface area (BSA) affected by psoriasis of patients included in the study was greater than $10 \%$, with a Psoriasis Area and Severity Index (PASI) greater than 12, and a static Physician's Global Assessment (sPGA) higher than 3. A total of 661 patients were randomized at baseline to receive brodalumab 140 or $210 \mathrm{mg}$ or a placebo (1:1:1 ratio). At the 12-week checkup, patients in the original brodalumab group who noted an improvement in SPGA were randomized again to receive induction doses of either brodalumab or placebo (1:1 ratio). Patients were differentiated by week-12 total body weight $(\leq 100 \mathrm{~kg} ;>100 \mathrm{~kg})$ and $\mathrm{sPGA}$ response $(0 ; 1)$. The endpoint - a PASI reduction of 75\% (PASI 75) — was based on the Phase II clinical trial (NCT00975637) and was 77\%, $82.5 \%$ and $10 \%$ of patients in the brodalumab 140 and $210 \mathrm{mg}$ and placebo groups, respectively; target sPGA success rates of $72 \%, 77 \%$, and $10 \%$ were the goal for the brodalumab 140 and $210 \mathrm{mg}$ and placebo groups, respectively. The percentage of patients who achieved PASI 75 and SPGA $0 / 1$ at the end of the 12-week induction phase was significantly higher for patients receiving brodalumab 140 or $210 \mathrm{mg}$ every 2 weeks (Q2W) compared to those receiving placebo. Significant differences between the brodalumab and placebo groups could even be seen 2 weeks after treatment began. PASI 75 was achieved by $83 \%, 60 \%$ and $3 \%$ of patients in the brodalumab $210 \mathrm{mg}$ Q2W, brodalumab $140 \mathrm{mg}$ Q2W, and placebo groups, respectively.

Percentage of patients who maintained PASI 90 and PASI 100 through week 52 of the withdrawal phase was respectively $78.3 \%$ and $67.5 \%$ for brodalumab at a dose of $210 \mathrm{mg}$ and $66.7 \%$ and $43.9 \%$ for brodalumab at a dose of $140 \mathrm{mg} .{ }^{14}$ Moreover, it is important to underline that $92.1 \%, 91.2 \%$, and $90.5 \%$, respectively, of the patients who exhibited PASI 75, 90, and 100 prior to brodalumab withdrawal reached their prior score in the first 16 weeks of retreatment. ${ }^{15}$

Here we report a retrospective analysis build on the cohort of patients treated with brodalumab coming from AMAGINE studies. 77 patients were receiving brodalumab when the study stopped in June 2015 at the sponsor's request: these were fifty males and twenty-seven female patients. The mean age was 48 years (range 22 to 75 years), the disease duration ranged from 6 to 60 years. 
Most patients had previously been exposed to systemic agents or biological therapy. The mean duration of treatment with brodalumab before discontinuation was 3.1 (Standard deviation (SD):1.5) years. The mean PASI score before initiation of brodalumab treatment was 20 (range 12-48). At the time of brodalumab treatment discontinuation, 67 patients $(87 \%)$ were clear or almost clear of psoriasis as defined by having reached PASI 90 to PASI 100 and 74 (96\%) had reached PASI 75 . The mean PASI at brodalumab discontinuation was 0.9 (range 0-19). All 77 (100\%) patients experienced a relapse of psoriasis within the 9 months follow-up period. The number (proportion) of patients who had not relapsed was $17(22 \%)$ at 3 months and $3(4 \%)$ patients at 6 months. The median time to relapse was 46 days, which is about 6.5 weeks (range 1 to 32 weeks). This retrospective analysis of the outcome of psoriasis in patients who had to stop brodalumab treatment shows that all patients relapsed within 9 months with a median time to relapse of 6 to 7 weeks. ${ }^{16}$

The PSSI (Psoriasis Scalp Severity Index) assesses the severity of scalp involvement of psoriasis along the parameters of erythema, induration and desquamation on a 5-point scale (0-4): absent, 0; slight, 1; moderate, 2; severe, 3 ; severest possible, 4 . The parameters are summed and multiplied by a value ranging between 0 to 6 that represents the area of the affected scalp. PSSI was valued across 12 weeks in the AMAGINE-1 trial. Brodalumab demonstrated efficacy in the treatment of scalp involvement in patients with moderate-to-severe plaque psoriasis across clinical subgroups defined by BMI, prior biologic treatment and baseline PSSI. Already at week 4, 40\% of patients receiving brodalumab $210 \mathrm{mg}$ Q2W with baseline PSSI $\geq 30$ had a PSSI 100 response. At week 8 of treatment with brodalumab $210 \mathrm{mg}$ Q2W, patients improved to a mean $\mathrm{SSA}<10 \%$, regardless of baseline PSSI. Patients with more severe scalp involvement achieved a similar rate of total clearance at week 12 compared to those with less severe involvement. ${ }^{17}$

AMAGINE-2 (NCT01708603) was a randomized, double-blind, placebo-controlled, parallel group multicenter trial. A total of 1,831 patients were randomly assigned in a $2: 2: 1: 1$ ratio to receive, respectively, either brodalumab $210 \mathrm{mg}$ or brodalumab $140 \mathrm{mg}$ (both injected subcutaneously on day 1 and at weeks 1, 2, 4, 6, 8 and 10), ustekinumab (injected subcutaneously, $45 \mathrm{mg}$ for patients with body weight $\leq 100 \mathrm{~kg}$ and $90 \mathrm{mg}$ for patients with body weight $>100 \mathrm{~kg}$, on day 1 , week 4 and every 12 weeks (Q12W) thereafter), or placebo (subcutaneous injection on day 1 and weeks 1, 2, 4, 6, 8 and 10). At week 12 , patients undergoing brodalumab treatment were randomized again in a $2: 2: 2: 1$ ratio to receive brodalumab $210 \mathrm{mg}$ Q2W, $140 \mathrm{mg}$ Q2W, $140 \mathrm{mg}$ every 4 weeks (Q4W), or $140 \mathrm{mg}$ every 8 weeks (Q8W), respectively, for 52 weeks. Patients originally assigned to the placebo group were switched to brodalumab $210 \mathrm{mg}$ Q2W from week 12 through 52. Those taking ustekinumab continued to follow the same treatment regime. The two-fold trial endpoint at 12 weeks consisted of PASI 75 and sPGA 0 or 1 for patients taking brodalumab versus placebo, and PASI 100 for patients taking brodalumab versus ustekinumab. Secondary endpoints consisted of PASI 90, PASI 100, and the psoriasis symptom inventory (PSI) response at week 12 and sPGA 0 or 1 at week 52. Brodalumab $210 \mathrm{mg}$ and brodalumab $140 \mathrm{mg}$ were shown to be statistically superior to placebo at 12 weeks. For the brodalumab $210 \mathrm{mg}$ and brodalumab $140 \mathrm{mg}$ groups, $86.3 \%$ and $66.6 \%$ of patients, respectively, achieved PASI 75 compared to $8.1 \%$ for the placebo group. PASI 100 response rates at week 12 were significantly higher with $210 \mathrm{mg}$ of brodalumab than with ustekinumab: $44 \%$ vs $22 \%$ respectevely $(P<0.001) .{ }^{18,19}$

It is important to underline that more than $90 \%$ of patients had a Dermatology Life Quality Index (DLQI) response by week 12, a response rate that continued through week 120, independent of the change in dose. At each point after open-label extension (OLE) baseline, a majority of patients reported a DLQI score of 0 , ie, psoriasis had "no effect at all on patient's life". 20

Like AMAGINE-2, the AMAGINE-3 study (NCT01708629) consisted of a randomized, double-blind, placebo-controlled, multicenter trial. A total of 1,881 patients were randomly distributed in a $2: 2: 1: 1$ ratio to receive, respectively, either brodalumab $210 \mathrm{mg}$ or brodalumab $140 \mathrm{mg}$ (injected subcutaneously on day 1 and at weeks 1 , 2, 4, 6, 8 and 10), ustekinumab (injected subcutaneously, $45 \mathrm{mg}$ for patients with body weight $\leq 100 \mathrm{~kg}$ and $90 \mathrm{mg}$ for patients with body weight $>100 \mathrm{~kg}$, on day 1 , at week 4 and every 12 weeks) or placebo injected subcutaneously on day 1 and at weeks 1, 2, 4, 6, 8 and 10. Brodalumab $210 \mathrm{mg}$ and brodalumab $140 \mathrm{mg}$ were shown to be statistically superior over placebo at 12 weeks. PASI 75 was achieved for $85.1 \%$ and $69.2 \%$ of patients for brodalumab $210 \mathrm{mg}$ and brodalumab $140 \mathrm{mg}$, respectively, compared to $6.0 \%$ of patients in the placebo group $(P<0.001$ compared to placebo). PASI 100 response rates at week 12 were significantly higher with $210 \mathrm{mg}$ of brodalumab than with ustekinumab, similarly to AMAGINE-2 ones: $37 \%$ vs $19 \%(P<0.001)$. 
New data from long-term, open-label extension studies of AMAGINE-2 and -3 trials have recently been published regarding the effectiveness of brodalumab to maintain clearance of psoriatic lesions beyond 52 weeks. At week 52, the rates of PASI 75, PASI 90 and PASI 100 were $93.1 \%, 86.4 \%$, and $66.5 \%$, respectively. The respective rates were $91.0 \%, 79.7 \%$, and $61.9 \%$ at week 108 Table 1.

At weeks 52 and 108, respectively, success rates based on $\mathrm{sPGA}$ score $\leq 1$ were $87.5 \%$ and $79.7 \% .{ }^{19}$ In Phase-III studies of brodalumab in patients with moderate-to-severe plaque psoriasis (AMAGINE-1, -2 and -3), PASI was positively correlated with DLQI regardless of treatment arm and brodalumab demonstrated superiority to ustekinumab in time to concurrent PASI 100 and DLQI 0. At week $28,50 \%$ of patients receiving brodalumab $210 \mathrm{mg}$ Q2W had achieved concurrent PASI 100 and DLQI 0 , but this response rate was not achieved in the ustekinumab group by week $52 .{ }^{21}$

Recent analyses, published in a letter by Blauvelt et al, were made on the achievement of sPGA success (score 0/1),
PASI 75 , and median times to achieve $25 \%, 50 \%$ and $75 \%$ reductions in baseline PASI in the AMAGINE- 2 and 3 trials. The estimated time required for $25 \%$ of patients to reach PASI 75 were 2.1 weeks for brodalumab and 4.8 weeks for ustekinumab. In comparison with corresponding times also for other treatments (2.4 weeks for ixekizumab, 3.0 weeks for high-dose secukinumab), brodalumab may act more quickly than any biologic therapy used in psoriasis. The rapid, prolonged effectiveness of brodalumab was also demonstrated by the improvement in baseline PASI over time. The time it took $50 \%$ of patients to reach PASI 75 was estimated at 4.2 weeks for brodalumab and 9.4 weeks for ustekinumab. ${ }^{22-25}$ The cumulative incidence of complete clearance over 52 weeks in AMAGINE-2 and -3 trials was analyzed using a competing risk model with outcomes of achieving PASI 100 or an inadequate response; and a static PGA $\geq 3$ or persistent values of 2 over at least a 4-week period starting at week 16 or later. In systemic/biologic-naïve patients, $76 \%$ receiving brodalumab achieved PASI 100 by week 52 compared to $58 \%$ of patients on ustekinumab. The percentage of patients achieving PASI 100 remained high for

Table I Comparing week 12 AMAGINE 2 and AMAGINE 3 PASI 75, 90 and 100 results with extension study (week 52 and week 108 results are obtained from patients assuming brodalumab $210 \mathrm{mg}$ Q2W during the maintenance and long-term extension phases)

\begin{tabular}{|c|c|c|c|}
\hline Week I 2 AMAGINE 2 & Week I 2 AMAGINE 3 & Week 52 AMAGINE 2-3 & $\begin{array}{l}\text { Week } 108 \text { AMAGINE } \\
2-3\end{array}$ \\
\hline \multicolumn{4}{|l|}{ PASI 75} \\
\hline $\begin{array}{l}\text { - } 66,6 \% \text { for brodalumab } 140 \mathrm{mg} \text { group } \\
\text { - } 86,3 \% \text { for brodalumab } 210 \mathrm{mg} \text { group } \\
\text { - } 70 \% \text { for ustekinumab group and } 8,1 \% \text { for } \\
\text { placebo group }\end{array}$ & $\begin{array}{l}\text { - } 69,2 \% \text { for brodalumab } 140 \\
\text { mg group } \\
\text { - } 85,1 \% \text { for brodalumab } 210 \\
\mathrm{mg} \text { group } \\
\text { - } 69,3 \% \text { for ustekinumab } \\
\text { group } \\
\text { - } \text { and } 6 \% \text { for placebo group }\end{array}$ & $\begin{array}{l}\text { - } 93,1 \% \text { for brodalumab } 210 \\
\text { mg group }\end{array}$ & $\begin{array}{l}\text { - } 91,0 \% \text { for brodalumab } \\
210 \mathrm{mg} \text { group }\end{array}$ \\
\hline \multicolumn{4}{|l|}{ PASI 90} \\
\hline & & $\begin{array}{l}\text { - } 86,4 \% \text { for brodalumab } 210 \\
\mathrm{mg} \text { group }\end{array}$ & $\begin{array}{l}\text { - } 79,7 \% \text { for } \\
\text { brodalumab } 210 \mathrm{mg} \\
\text { group }\end{array}$ \\
\hline \multicolumn{4}{|l|}{ PASI 100} \\
\hline $\begin{array}{l}\text { - } 25,7 \% \text { for brodalumab } 140 \mathrm{mg} \text { group } \\
\text { - } 44,4 \% \text { for brodalumab } 210 \mathrm{mg} \text { group } \\
\text { - } 21,7 \% \text { for ustekinumab group, } \\
\text { - } \text { and } 0,6 \% \text { for placebo group }\end{array}$ & $\begin{array}{l}\text { - } 27 \% \text { for brodalumab } 140 \mathrm{mg} \\
\text { group } \\
\text { - } 36,7 \% \text { for brodalumab } 210 \\
\mathrm{mg} \text { group } \\
\text { - } 18,5 \% \text { for ustekinumab } \\
\text { group } \\
\text { - } \text { and } 0,3 \% \text { for placebo group }\end{array}$ & $\begin{array}{l}\text { - } 66,5 \% \text { for brodalumab } 210 \\
\text { mg group }\end{array}$ & $\begin{array}{l}\text { - } 61,9 \% \text { for } \\
\text { brodalumab } 210 \mathrm{mg} \\
\text { group }\end{array}$ \\
\hline
\end{tabular}

Notes: Data from Lebwohl $M$ et al ${ }^{18}$ and Papp KA et al. ${ }^{28}$ 
brodalumab in systemic use, biologic-naïve (78\%), biologic use without failure (75\%) and biologic failure (70\%) patients, but were lower for ustekinumab in systemic use, biologicnaïve (55\%) and biologic use without failure (41\%) patients. The percentages were lower still for ustekinumab patients with biologic failure $(30 \%)$. These data show that patients with moderate-to-severe plaque psoriasis treated with brodalumab achieved complete clearance more rapidly and more frequently than patients treated with ustekinumab, independent of treatment history. ${ }^{26}$

One interesting report evaluated PSI response across AMAGINE-1/-2/-3 trials. In all three studies, a higher percentage of patients achieved the secondary endpoint (PSI total score $\leq 8$ with no item score $>1$ ) at week 12 with brodalumab $140 \mathrm{mg}$ Q2W (range, 51\%-53\%) and $210 \mathrm{mg} \mathrm{Q} 2 \mathrm{~W}$ (range, 61\%-68\%) compared to placebo (range, 4\%-7\%). In AMAGINE-2/-3, the studies with an active comparator (ustekinumab), the percentage of patients with a PSI total score response was higher for brodalumab $210 \mathrm{mg}$ Q2W (range, 61\%-68\%) than both brodalumab $140 \mathrm{mg}$ Q2W (range, $51 \%-53 \%$ ) and ustekinumab (range, $52 \%-55 \%$ ). ${ }^{27}$ For the same trials at week 12, the brodalumab $210 \mathrm{mg}$ group contained many more patients with a PSI score of $0(22.7 \%)$ compared to ustekinumab $(13.4 \% ; P<0.001)$. The median time to PSI total score response was estimated to be 4 weeks for brodalumab $210 \mathrm{mg}$ and 5 weeks for brodalumab $140 \mathrm{mg}$; it was not estimated for placebo. As measured from baseline, the median time to achieve $\geq 50 \%$ improvement in PSI total score was estimated to be 2.6 weeks for brodalumab $210 \mathrm{mg}$ and 3 weeks for brodalumab $140 \mathrm{mg}$; no estimate was made for placebo. Analyzing psoriasis symptoms by integrating different indicators (PASI, SPGA, PSI) highlights a decrease in symptoms captured by the PSI and the clinical severity of lesions captured by PASI among patients who received 12 weeks of brodalumab $210 \mathrm{mg}$ Q2W compared to placebo. ${ }^{27}$

To date, there have been six Phase-II and four PhaseIII RCTs that have assessed the efficacy and safety of brodalumab in treating psoriasis, psoriatic arthritis, Crohn's disease, asthma and rheumatoid arthritis.14,18,28-34 All studies had a 12-week induction period where patients received brodalumab on day 1 and at week 1 , week 2 , and every other week up to week 10 , with evaluation at week 12 . Six of the studies continued with an open-label extension offering brodalumab to all patients. Adverse event data is currently available for open-label extensions up to week 52; however, the Phase-II psoriasis study by Papp et al $^{14}$ has data available up to week $120 .^{20}$
Targan et $\mathrm{al}^{30}$ terminated their study prematurely as brodalumab was found to worsen the activity of Crohn's disease. During the 12-week induction period within the study, 4,118 patients were treated with at least one dose of brodalumab. During induction, the most common adverse events reported with brodalumab use were nasopharyngitis (7.4\%), upper respiratory tract infection $(5.6 \%)$, headaches $(4.3 \%)$, arthralgias $(3.0 \%)$, and injection site reaction $(2.1 \%)$. Due to the immunosuppressive nature of brodalumab, serious infections, candidiasis, and neutropenia are considered adverse events of interest. Only $1.7 \%$ of brodalumab patients reported serious infections, similar to the placebo group (1.5\%). Candidiasis was reported in $1.1 \%$ of patients, typically oropharyngeal, and $0.5 \%$ were found to have clinically insignificant neutropenia. Major adverse cardiovascular events, headache, fatigue, diarrhea, nausea, and vomiting were reported by less than $1 \%$ of participants. Similar to a study with another IL-17 antagonist, secukinumab, Targan et al, ${ }^{30}$ discovered worsening of Crohn's disease activity with IL-17 inhibition in 24 (18\%) of 130 patients, and the study was therefore terminated early. Subsequent RCTs in other states of disease excluded subjects with a history of Crohn's disease.

A total of 4,340 patients received brodalumab for an extended duration of 52 to 120 weeks. Adverse event data was compiled from six open-label extensions and a 52-week study by Yamasaki and colleagues. ${ }^{34}$ The most common adverse events reported with longer duration of brodalumab therapy were injection site reaction (4.5\%), candida infections (4.1\%), and nasopharyngitis (3\%). Other side effects included upper respiratory tract infection $(1.8 \%)$, serious infection (1.4\%), arthralgias (1.1\%), and neutropenia (1.1\%). Headache, fatigue, major adverse cardiovascular events, and gastrointestinal adverse events (eg, nausea, vomiting and diarrhea) were reported by less than $1 \%$ of patients. Depressed mood was described by only $1.5 \%$ of brodalumab patients, similar to the $1.3 \%$ of participants receiving ustekinumab during the AMAGINE-2 and -3 trials. ${ }^{18}$ All brodalumab clinical trials were stopped on 22 May 2015, as six participants treated with brodalumab across all programs committed suicide. Of these six, four were enrolled in psoriasis studies, one in the rheumatoid arthritis trial, and one in a psoriatic arthritis study. ${ }^{17}$ Of the four psoriasis patients, two committed suicide during the open-label extension of AMAGINE-2 and the remaining two occurred during the open-label extension of AMAGINE-1, one of which was deemed to be an unintentional heroin overdose on autopsy. One suicide was committed during the induction period of the 
Table 2 Safety data from AMAGINE-2 and AMAGINE-3 trials

\begin{tabular}{|c|c|c|}
\hline Study protocols & Most common AE & Severe AEs \\
\hline Study protocols & $\begin{array}{l}\text { Most common AEs (rates per } 100 \\
\text { patient-over } 2.3 \text { years) }\end{array}$ & Severe AEs \\
\hline \multicolumn{3}{|l|}{ AMAGINE-2 } \\
\hline $\begin{array}{l}\mathrm{N}=1800 \text { patients with moderate-to-severe plaque } \\
\text { psoriasis } \\
\text { Placebo Q2W, Brodalumab Q2W } 140 \mathrm{mg} \\
\text { Brodalumab Q2W } 210 \mathrm{mg} \\
\text { Ustekinumab as per label } \\
\text { Follow up } 52 \text { weeks, extended to } 120 \text { weeks }\end{array}$ & $\begin{array}{l}\text { - Injection site reaction (4.5\%) } \\
\text { - Candida infections (4.1\%) } \\
\text { - Nasopharyngitis (3\%) }\end{array}$ & $\begin{array}{l}\text { - } 4 \text { patients committed suicide } \\
\text { - Neutropenia (0.2 for AMAGINE-2 and } 1.5 \text { for } \\
\text { AMAGINE-3) } \\
\text { - Sepsis }(0.1),(0.1)\end{array}$ \\
\hline \multicolumn{3}{|l|}{ AMAGINE-3 } \\
\hline $\begin{array}{l}\mathrm{N}=1800 \text { patients with moderate-to-severe plaque } \\
\text { psoriasis } \\
\text { Placebo Q2W } \\
\text { Brodalumab Q2W } 140 \mathrm{mg} \\
\text { Brodalumab Q2W } 210 \mathrm{mg} \\
\text { Ustekinumab as per label } \\
\text { Follow up } 52 \text { weeks, extended to } 120 \text { weeks }\end{array}$ & $\begin{array}{l}\text { - Respiratory tract infection } \\
(1.8 \%) \\
\text { - Arthralgias ( } 1.1 \%) \\
\text { - Depressed mood (I.5\%) }\end{array}$ & $\begin{array}{l}\text { - MACE }(0.2),(0.7) \\
\text { - Serious infection (1.4\%) }\end{array}$ \\
\hline
\end{tabular}

rheumatoid arthritis study by Pavelka et al, ${ }^{29}$ and one during the AMVISION-1 open-label extension. The FDA conducted a thorough analysis of the data available in 2015 and determined that there was no established drug-related risk of suicide or suicidal ideation Table 2.

Suicidal behavior related to use of the IL-17 receptor antagonist was unexpected, and there is currently no known biological explanation why brodalumab would generate psychiatric adverse events; further investigation is therefore needed regarding such possibilities. However, the explanation for increased suicidal behavior may be lie in a confounding effect. At the time the AMAGINE studies were carried out, suicide rates increased in the United States, Canada and Poland, and in white men over 45 years of age in particular. The majority of patients included in the AMAGINE studies were middle-aged white men, which may explain the suicide rates in the AMAGINE trials. As described by Danesh et al, this increase in suicide among white men may also have created a confounding effect in other psoriasis studies with similar patient demographics. For example, between 2010 and 2015, the effect of apremilast on psoriasis and psoriatic arthritis was studied in phase-III trials in which an increased risk of depression and suicidal ideation was also identified. ${ }^{35-37}$

Late-stage clinical trials of brodalumab in psoriasis patients were explored to identify the occurrence of psychiatric adverse events and to search for possible causal relationships with the drug treatment under study. Data related to psychiatric adverse events were aggregated from 5 clinical trials: a Phase-II, randomized, double-blinded, placebo-controlled, dose-ranging study (ClinicalTrials.gov identifier NCT00975637); an openlabel, long-term extension of the Phase-II study (NCT01101100); and three Phase-III, randomized, doubleblind, controlled trials (AMAGINE-1, -2 and -3 [NCT01708590, NCT01708603 and NCT01708629, respectively]) along with their long-term open-label extensions. All five studies monitored various psychiatric adverse events throughout the treatment and follow-up periods using the Columbia Suicide Severity Rating Scale (C-SSRS), the Patient Health Questionnaire-8 and the Hospital Anxiety and Depression Scale (HADS) questionnaire. In addition, the Columbia Classification Algorithm for Suicide Assessment (C-CASA) was used for an independent judgement about suicidal ideation and behavior (SIB) events. ${ }^{38-41}$ The results showed that the occurrence of SIB events during treatment with brodalumab was similar to the rate for placebo or ustekinumab during the induction phase and comparable to the rate for ustekinumab during the first year of treatment. The rate of psychiatric adverse events in patients receiving brodalumab was similar to the rate in patients receiving placebo for 12 weeks and in patients receiving ustekinumab for 12 and 52 weeks. No association was found between the inhibition of IL-17 signaling and pathogenic mechanisms related to neuropsychiatric adverse events. In sum, no evidence of causality was 
found from the inquiry into SIB events among psoriasis patients in the brodalumab trials.

The most relevant data for investigating a potential causal relationship between brodalumab and SIB events are found in the controlled sections of the Phase-II and -III trials due to the presence of a group of patients with similar baseline characteristics assigned to a comparator and the same evaluation techniques used across multiple cohorts. During the period of controlled study, no increases in SIB rates were observed in patients treated with brodalumab compared to patients treated with placebo or ustekinumab. ${ }^{42}$

\section{Discussion about the safety of brodalumab}

SIB events found in brodalumab trials are one of the most challenging aspects with respect to safety. In a letter, Rieder underlines parallels between brodalumab and isotretinoin. From his point of view, both isotretinoin and brodalumab are highly effective medications developed for patients who are dermatologically and often psychiatrically ill. Both have also experienced controversial associations with SIB events. ${ }^{43}$ Several recent studies have investigated possible relationships between psoriasis and psychiatric diseases. A populationbased study from the National Health and Nutrition Examination Survey examined the association between psoriasis and major depression in 12,382 US citizens. Among patients with self-reported psoriasis $(2.8 \%$ of the study population), $16.5 \%$ met the criteria for major depression, which was more than double the $7.8 \%$ prevalence found in the general study population. Even after controlling for various demographic and medical characteristics (sex, age, race, body mass index (BMI), physical activity, smoking history, alcohol use, and a history of myocardial infarction, stroke or diabetes mellitus), psoriasis was still significantly associated with major depression (OR 2.09, 95\% CI 1.41-3.11). ${ }^{44}$ Investigations have increasingly focused on the role of the immune system and systemic inflammation in the pathophysiology of depression, although the exact role of IL-17 in depression is still unclear. One study in patients with rheumatoid arthritis examined the levels of IL-17 among those with or without comorbid depression or anxiety. Serum levels were significantly higher in patients with both rheumatoid arthritis and anxiety compared to patients without anxiety $(P=0.044)$. In addition, IL-17 levels were positively correlated with the severity of anxiety, even after controlling for the severity of pain and arthritis disease. In contrast, a study examining IL-17 and IL-23 plasma levels in patients with major depressive disorder found no difference in these markers relative to healthy controls. ${ }^{45,46}$ Further investigation is needed to evaluate the direct role played by IL-17 in pathological conditions of the central nervous system. In the case of brodalumab, several factors should be considered regarding the problem of SIB comorbidities, including a clinical trial design that did not exclude patients based on psychiatric history, the lack of increased SIB events during periods when brodalumab was actively compared to placebo or ustekinumab, and the known risk of SIB events in the psoriasis population.

The numerical imbalance of completed suicides seen in the brodalumab psoriasis studies warranted a boxed warning regarding possible increases in SIB on the US FDAapproved label, though the same label states there is no causal association between brodalumab exposure and suicide. $^{47,48}$

In addition, the FDA's decision to insert such a warning was heavily influenced by the fact that SIB behavior in the brodalumab trial manifested as completed suicides, despite the presence of attempted suicides in other biologic trials. On the other hand, in Europe, after EMA revision and drug approval, there is no black box warning.

Continued research is needed in order to identify vulnerable patients and to better assess risk versus benefit on the level of the individual. Importantly, the role of the physician in remaining vigilant of SIB events in psoriasis patients should not be limited to solely biologic therapies or boxed warnings.

With regard to other adverse events reported with brodalumab the safety profile of brodalumab overall is consistent with other IL-17-blocking drugs and broadly consistent with the spectrum of adverse events seen with biologic agents used to treat moderate-to-severe plaque psoriasis. ${ }^{48}$

\section{Brodalumab, the latest approved treatment in the anti-IL- 17 class: what will make it different in real life?}

The great advances made in our understanding of T-cell biology recently have led to the development of remarkably successful anti-IL-17 medications. The IL-17/IL-23 axis is now known to influence psoriasis, and new innovative treatments that inhibit IL-17 mark a sea change in the medical management of psoriasis patients, with a large portion of patients with moderate-to-severe psoriasis achieving PASI 90-100. 
Brodalumab provides an important new therapy for the management of psoriasis, because there remains a significant unmet patient need for new agents that can provide novel mechanisms of action, a rapid onset of effect, improved and sustained total skin clearance, greater compliance, and the minimization of drug-specific safety concerns.

In sum, brodalumab $210 \mathrm{mg}$ Q2W met all primary endpoints across all Phase-III studies, demonstrating short and long-term efficacy in the treatment of psoriasis. Approximately twice as many patients who received brodalumab $210 \mathrm{mg}$ Q2W achieved total skin clearance as measured by PASI 100, compared to ustekinumab at weeks 12 and 52 . More than $50 \%$ of patients on brodalumab $210 \mathrm{mg}$ Q2W were completely clear of their disease (PASI 100) within a year of initiating treatment.

On the other hand, in recent months, a paradoxical eczematous reaction during treatment with ixekizumab ${ }^{49}$ and a report of atopic-like dermatitis after secukinumab injection ${ }^{50}$ have been reported in the literature. In our experience, we have observed similar cases in our daily practice.

As indicated by the data on targeted therapeutics, both Th2 and Th22 seem to influence atopic dermatitis (AD), with variable Th1 and Th17 activation. ${ }^{51,52}$ However, while psoriasis appears to be relatively homogenous and centered on inflammation driven by Th17/IL-23, AD seems to be heterogeneous over different phenotypes, possibly reflecting interactions between Th2 cytokines and different levels of contributions of IL-17, IL-22 and other cytokines. For example, some AD phenotypes (eg, Asian $\mathrm{AD}$, intrinsic $\mathrm{AD}$ and pediatric $\mathrm{AD}$ ), while sharing Th2 activation, show higher IL-17 expression and histologic features like those seen in psoriasis. ${ }^{53-55}$ The evidence suggests that in both $\mathrm{AD}$ and psoriasis, IL-17C overproduction may drive feed-forward inflammation, including influencing the S100A7-A9 proteins that accompany psoriasiform hyperplasia.

IL-17C, which is produced by epidermal keratinocytes and other non-immune cell types, signals through receptor subunits IL-17RA and IL-17RE. These are expressed in various types of cells, including keratinocytes and $\mathrm{T}$ lymphocytes, so IL-17C participates in both autocrine signaling in the epidermis and paracrine signaling to immune cells.

Due to the simulation of Th17 T cells by IL-17C, the synthesis of IL-17A/F (products of activated lymphocytes) and IL-22 increase and can thereby clearly promote autoimmunity. ${ }^{54}$ IL-17A and IL-17C are involved in a mutual relationship wherein IL-17A strongly induces
IL-17C in keratinocytes and IL-17C is capable of synthesizing IL-17A in T lymphocytes. Large amounts of IL-17C are produced in keratinocytes (ie, production in psoriasis is about 100 times greater than IL-17A), and the actions of this cytokine may thus dominate the IL-17 response axis in chronic inflammatory conditions.

By locally amplifying skin inflammation, IL-17C may also play a role in other skin inflammatory diseases; inhibiting IL-17C may therefore be beneficial in both psoriasis and AD patients: antibody-dependent blockade of IL-17C inhibited cutaneous inflammation in the IL-23-induced psoriasis model and even in AD-like inflammation in mice $^{56-59}$ To date, brodalumab is the only anti-IL-17 treatment that affects not only IL-17A and IL-17F, but also IL-17C, since it blocks the shared IL-17 receptor A. In this sense, brodalumab could have beneficial effects not only for psoriasis but also for atopic dermatitis. It may also represent a therapeutic alternative in patients who develop paradoxical eczematous reaction or atopic-like dermatitis during treatment with other anti-IL-17A therapies (secukinumab, ixekizumab) Figure 1.

In the new era of anti-IL23p19 drugs, we are unable to state whether these will be superior to anti-IL17 treatments in reality. Some scientists suggest there are advantages to blocking IL-17 instead of IL-23. This is because several pathways converge towards a unique cytokine: IL-17. Furthermore, by blocking IL-17, not only IL-23 induced cytokine is neutralized, but also the one induced by IL-1 $\beta$,

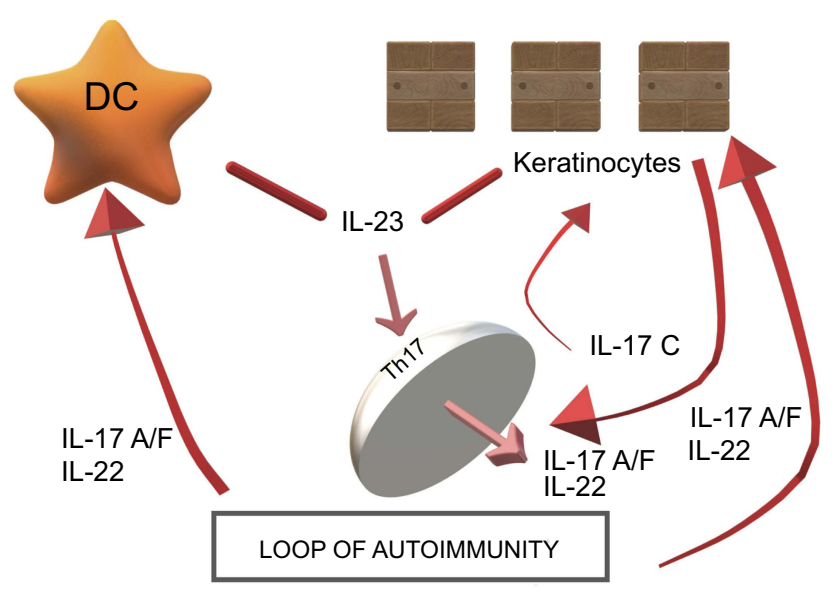

Figure I IL-17A and IL-I7C are involved in a mutual relationship wherein IL-I7A strongly induces IL-I7C in keratinocytes and IL-I7C is capable of synthesizing IL17A in T lymphocytes. IL-I7C may also play a role in other skin inflammatory diseases; inhibiting IL-I7C may therefore be beneficial in both psoriasis and AD patients: antibody-dependent blockade of IL-I7C inhibited cutaneous inflammation in the IL-23-induced psoriasis model and even in AD-like inflammation in mice. Abbreviations: AD, atopic dermatitis; DC, dendritic cell; IL, interleukin; Th, lymphocyte $\mathrm{T}$ helper. 
and by blocking IL-17, proteic autoantigen production and AMP, and thus the activation of pDCs, are inhibited. Other scientists claim it may be more beneficial to reduce Th17 clonal expansion with IL-23 blockers, one potential benefit of which is the low dosing frequency and a sustained drug effect. Inhibition of IL-23 may be more effective and the effects longer-lasting due to an upstream effect in which the expression of several downstream pro-inflammatory cytokines secreted by Th17 cells (eg IL-17A, IL-17F, IL21 and IL-22) is reduced. One possible explanation is impaired survival or a phenotype change in pathogenic Th17 cells, or the restoration of altered T-reg function. This is important since Th17 cells are long-lived and metabolically active, even after the skin is healed. It could therefore be beneficial to inhibit the initial production of these cells. Due to its broader mechanism of action, brodalumab could certainly be superior to the anti-IL17A treatment, with an efficacy more similar to anti-IL23p19 therapies.

The availability of a variety of psoriasis treatment options could enable the best treatment to be chosen for each patient in view of factors such as comorbidities (hypertension, liver disorders, and cardiovascular diseases), patient age and patient needs (work activities, social life, fear/anxiety of injections, etc.). As well, psoriasis is a chronic condition and wider array of therapies would also allow psoriasis to be managed more safely, effectively and satisfactorily, not only for an immediate response, but also in the long term. Psoriasis consists of several clinically similar diseases that are genetically different and likely operate under different pathogenetic mechanisms, so it is to be expected that varied responses to therapies are seen in both the short and long terms.

Despite the increase in therapeutic alternatives to treat psoriasis, some unanswered questions remain, such as the side-effect profile, immunogenicity, and initial lack or later loss of efficacy. The effectiveness of one biologic is not necessarily determined by the failure of another, and clinicians must be ready to quickly modify the treatment if the clinical goals remain unmet. Indeed, these drugs have a relatively high diminishing response to treatment: between $10 \%$ and $25 \%$ of patients report loss of efficacy. Significant expenses can potentially be avoided by reducing the number of failed treatments with biologics. Precision medicine and pharmacogenomics are therefore the targets of much research to uncover biomarkers that can guide clinicians when prescribing treatments, allowing them to predict the likelihood of efficacy and side effects and thereby shifting into an era of real tailored, patientcentered therapy. ${ }^{60-63}$

In this context, stratified medicine can potentially optimize psoriasis management. The response of psoriasis patients to different drugs should be compared in clinical practice and various clinical, genetic, and immune biomarkers should be identified to predict said responses, thereby leading to tailored therapy, the new goal for biologic drugs in psoriasis.

\section{Disclosure}

The authors report no conflicts of interest in this work.

\section{References}

1. World Health Organization. Global Report on Psoriasis. Geneva: World Health Organization; 2016.

2. Strohal R, Kirby B, Puig L, et al. Psoriasis beyond the skin: an expert group consensus on the management of psoriatic arthritis and common co-morbidities in patients with moderate-to-severe-psoriasis. $J$ Eur Acad Dermatol Venereol. 2014;28(12):1661-1669. doi:10.1111/ jdv.2014.28.issue-12

3. Talamonti M, Galluzzo M, Servoli S, D’Adamio S, Bianchi L. Alexithymia and plaque psoriasis: preliminary investigation in a clinical sample of 250 patients. Dermatology. 2016;232(6):648-654. doi: $10.1159 / 000453661$

4. Oliveira Mde F, Rocha Bde O, Duarte GV. Psoriasis: classical and emerging comorbidities. An Bras Dermatol. 2015;90(1):9-20. doi:10.1590/abd1806-4841.20153038

5. American Academy of Dermatology Work Group; Menter A, Korman NJ, et al. Guidelines of care for the management of psoriasis and psoriatic arthritis: section 6. Guidelines of care for the treatment of psoriasis and psoriatic arthritis: case-based presentations and evidence-based conclusions. J Am Acad Dermatol. 2011;65(1):137-174. doi:10.1016/j.jaad.2010.11.055

6. Griffiths CE, Barker JN. Pathogenesis and clinical features of psoriasis. Lancet. 2007;370(9583):263-271. doi:10.1016/S0140-6736(07) 61128-3

7. Warren RB. Efficacy and safety of emerging immunotherapies in psoriasis. Immunotherapy. 2015;7(2):119-133. doi:10.2217/ imt.14.101

8. Schadler ED, Ortel B, Mehlis SL. Biologics for the primary care physician: review and treatment of psoriasis. Dis Mon. 2019;65 (3):51-90.

9. Lowes MA, Kikuchi T, Fuentes-Duculan J, et al. Psoriasis vulgaris lesions contain discrete populations of Th1 and Th17 T cells. $J$ Investig Dermatol. 2008;128(5):1207-1211. doi:10.1038/sj. jid.5701213

10. Marinoni B, Ceribelli A, Massarotti MS, Selmi C. The Th17 axis in psoriatic disease: pathogenetic and therapeutic implications. Auto Immun Highlights. 2014;5(1):9-19. doi:10.1007/s13317-013-0057-4

11. Chang SH, Dong C. A novel heterodimeric cytokine consisting of IL17 and IL-17F regulates inflammatory responses. Cell Res. 2007;17:435-440. doi:10.1038/cr.2007.35

12. Carrascosa JM, Jacobs I, Petersel D, Strohal R. Biosimilar drugs for psoriasis: principles, present, and near future. Dermatol Ther (Heidelb). 2018;8(2):173-194. doi:10.1007/s13555-018-0230-9

13. European Medicines Agency. Summary of Product Characteristics: Kyntheum $210 \mathrm{mg}$ Solution for Injection in Pre-Filled Syringe; 2017. Available from: http://www.ema.europa.eu. Accessed February 27, 2018. 
14. Papp KA, Reich K, Paul C, et al. A prospective phase 3, randomized, double-blind, placebo-controlled study of brodalumab in patients with moderate-to severe plaque psoriasis. Br J Dermatol. 2016;175 (2):273-286. doi:10.1111/bjd.14493

15. Armstrong A, Blauvelt A, Wu JJ et al. Retreatment with brodalumab results in high response rates in patients with psoriasis after treatment interruption. Abstract from 76th Annual Meeting of the American Academy of Dermatology; February 16-20; 2018; San Diego, CA.

16. Masson Regnault M, Konstantinou MP, Khemis A, et al. Early relapse of psoriasis after stopping brodalumab: a retrospective cohort study in 77 patients. J Eur Acad Dermatol Venereol. 2017;31 (9):1491-1496. doi:10.1111/jdv.14387

17. Philipp S, Hansen JB, Schultz Nielsen V et al. Long-term efficacy of brodalumab for the treatment of moderateto-severe psoriasis in two pivotal phase 3 clinical trials. Abstract from 27th EADV Congress; September 12-16; 2018; Paris, France.

18. Lebwohl M, Strober B, Menter A, et al. Phase 3 studies comparing brodalumab with ustekinumab in psoriasis. $N$ Engl J Med. 2015;373 (14):1318-1328. doi:10.1056/NEJMoa1503824

19. Lebwohl M, Papp KA, Tyring S et al. Long-term efficacy of brodalumab for the treatment of moderate-to-severe psoriasis in two pivotal phase 3 clinical trials. Abstract from 27th EADV Congress; September 12-16; 2018; Paris, France.

20. Papp K, Leonardi C, Menter A, et al. Safety and efficacy of brodalumab for psoriasis after 120 weeks of treatment. $\mathrm{J} \mathrm{Am} \mathrm{Acad}$ Dermatol. 2014;71(6):1183-1190.e3. doi:10.1016/j.jaad.2014.08.039

21. Lambert J, Hansen BJ, Nielsen V et al. Correlation between psoriasis area and severity index and dermatology life quality index in patients with moderate to severe psoriasis treated with brodalumab in the AMAGINE studies. Abstract from 27th EADV Congress; September 12-16; 2018; Paris, France.

22. Blauvelt A, Papp KA, Lebwohl MG, et al. Rapid onset of action in patients with moderate-to-severe psoriasis treated with brodalumab: A pooled analysis of data from two phase 3 randomized clinical trials (AMAGINE-2 and AMAGINE-3). J Am Acad Dermatol. 2017;77 (2):372-374. doi:10.1016/j.jaad.2017.03.026

23. Griffiths CEM, Reich K, Lebwohl M, et al. Comparison of ixekizumab with etanercept or placebo in moderateto-severe psoriasis (UNCOVER-2 and UNCOVER-3): results from two phase 3 randomised trials. Lancet. 2015;386(9993):541-551. doi:10.1016/S01406736(15)60125-8

24. Langley RG, Elewski BE, Lebwohl M. Secukinumab in plaque psoriasis: results of two phase 3 trials. $N$ Engl J Med. 2014;371 (4):326-338. doi:10.1056/NEJMoa1314258

25. Leonardi C, Langley R, Blauvelt A, et al. Rapid onset of efficacy in patients with psoriasis treated with ixekizumab: a pooled analysis of data from two randomized clinical trials (UNCOVER-2 and UNCOVER-3). Poster Presented at Am Acad Dermatol Annual Meeting; March; 2016; Washington, DC.

26. Reich K, Nielsen V, Hansen JB et al. Achieving complete clearance in the AMAGINE-2 and -3 studies: complete clearance over time in patients with moderate to severe plaque psoriasis treated with brodalumab up to 52 weeks. Abstract from 27th EADV Congress; September 12-16; 2018; Paris, France.

27. Gottlieb $\mathrm{AB}$, Gordon $\mathrm{K}$, Hsu S, et al. Improvement in itch and other psoriasis symptoms with brodalumab in phase 3 randomized controlled trials. J Eur Acad Dermatol Venereol. 2018. doi:10.1111/jdv.14913

28. Papp KA, Leonardi C, Menter A, et al. Brodalumab, an anti-interleukin-17- receptor antibody for psoriasis. $N$ Engl J Med. 2012;366 (13):1181-1189. doi:10.1056/NEJMoa1109017

29. Pavelka K, Chon Y, Newmark R, Lin S-L, Baumgartner S, Erondu N. A study to evaluate the safety, tolerability, and efficacy of brodalumab in subjects with rheumatoid arthritis and an inadequate response to methotrexate. J Rheumatol. 2015;42(6):912-919. doi:10.3899/ jrheum. 141271
30. Targan SR, Feagan B, Vermeire S, et al. A randomized, double-blind, placebo controlled phase 2 study of brodalumab in patients with moderate-to-severe Crohn's disease. Am J Gastroenterol. 2016;111 (11):1599-1607. doi:10.1038/ajg.2016.298

31. Mease PJ, Genovese MC, Greenwald MW, et al. Brodalumab, an anti-IL17RA monoclonal antibody, in psoriatic arthritis. $N$ Engl $J$ Med. 2014;370(24):2295-2306. doi:10.1056/NEJMoa1 315231

32. Nakagawa H, Niiro H, Ootaki K; Japanese Brodalumab Study Group. Brodalumab, a human anti-interleukin-17-receptor antibody in the treatment of Japanese patients with moderate-to-severe plaque psoriasis: efficacy and safety results from a phase II randomized controlled study. J Dermatol Sci. 2016;81(1):44-52. doi:10.1016/j. jdermsci.2015.10.009

33. Busse WW, Holgate S, Kerwin E, et al. Randomized, double-blind, placebocontrolled study of brodalumab, a human anti-IL-17 receptor monoclonal antibody, in moderate to severe asthma. Am J Respir Crit Care Med. 2013;188(11):1294-1302. doi:10.1164/rccm.201212$2318 \mathrm{OC}$

34. Yamasaki K, Nakagawa H, Kubo Y, Ootaki K; Japanese Brodalumab Study Group. Efficacy and safety of brodalumab in patients with generalized pustular psoriasis and psoriatic erythroderma: results from a 52-week, open-label study. Br $J$ Dermatol. 2017;176 (3):741-751. doi:10.1111/bjd.14702

35. Danesh MJ, Kimball AB. Brodalumab and suicidal ideation in the context of a recent economic crisis in the United States. $J \mathrm{Am}$ Acad Dermatol. 2016;74(1):190-192. doi:10.1016/j.jaad.2015. 08.057

36. Celgene Corporation. Highlights of Prescribing Information. Available from: http://www.otezla.com/otezla-prescribinginforma tion.pdf. Accessed July 5, 2018.

37. Galluzzo M, D'adamio S, Bianchi L, Talamonti M. Brodalumab for the treatment of psoriasis. Expert Rev Clin Immunol. 2016;12 (12):1255-1271. doi:10.1080/1744666X.2016.1246957

38. Lebwohl MG, Papp KA, Marangell LB, et al. Psychiatric adverse events during treatment with brodalumab: analysis of psoriasis clinical trials. J Am Acad Dermatol. 2018;78(1):81-89.e5. doi:10.1016/j. jaad.2017.08.024

39. Mundt JC, Greist JH, Gelenberg AJ, Katzelnick DJ, Jefferson JW, Modell JG. Feasibility and validation of a computer-automated Columbia-suicide severity rating scale using interactive voice response technology. J Psychiatr Res. 2010;44(16):1224-1228. doi:10.1016/j.jpsychires.2010.04.025

40. Kroenke K, Strine TW, Spitzer RL, et al. The PHQ-8 as a measure of current depression in the general population. $J$ Affect Disord. 2009;114(1-3):163-173. doi:10.1016/j.jad.2008.06.026

41. Posner K, Oquendo MA, Gould M, Stanley B, Davies M. Columbia Classification Algorithm of Suicide Assessment (C-CASA): classification of suicidal events in the FDA's pediatric suicidal risk. Am J Psychiatry. 2007;164:1035-1043. doi:10.1176/ajp.2007.164.7.1035

42. Chiricozzi A, Romanelli M, Saraceno R, Torres T. No meaningful association between suicidal behavior and the use of IL-17A-neutralizing or IL-17RA-blocking agents. Expert Opin Drug Saf. 2016;15 (12):1653-1659. doi:10.1080/14740338.2016.1228872

43. Rieder EA. In response to Lebwohl et al, "Psychiatric adverse events during treatment with brodalumab: analysis of psoriasis clinical trials". J Am Acad Dermatol. 2018;78(3):e73. doi:10.1016/j. jaad.2017.10.052

44. Cohen BE, Martires KJ, Ho RS. Psoriasis and the risk of depression in the US population: national health and nutrition examination survey 2009-2012. JAMA Dermatol. 2016;152(1):73-79. doi:10.1001/jamadermatol.2015.3605

45. Liu Y, Ho RC, Mak A. The role of interleukin (IL)-17 in anxiety and depression of patients with rheumatoid arthritis. Int $J$ Rheum Dis. 2012;15(2):183-187. doi:10.1111/j.1756-185X.2011.01673.x 
46. Kim JW, Kim YK, Hwang JA, et al. Plasma levels of IL-23 and IL-17 before and after antidepressant treatment in patients with major depressive disorder. Psychiatry Investig. 2013;10(3):294-299. doi:10.4306/pi.2013.10.3.294

47. Siliq (brodalumab) [package insert]. Bridgewater, NJ: Valeant Pharmaceuticals North America LLC; February 2017.

48. Rusta-Sallehy S, Gooderham M, Brodalumab: PK. A review of safety. Skin Therapy Lett. 2018;23(2):1-3.

49. Munera-Campos M, Ballesca F, Richarz N, et al. Paradoxical eczematous reaction to ixekizumab. J Eur Acad Dermatol Venereol. 2018. doi: $10.1111 /$ jdv. 15156

50. Burlando M, Cozzani E, Russo R, et al. Atopic-like dermatitis after Secukinumab injection: a case report. Dermatol Ther. 2018;20: e12751. doi:10.1111/dth.12751

51. Guttman-Yassky E, Brunner PM, Neumann AU, et al. Efficacy and safety of fezakinumab (an anti-IL-22 monoclonal antibody) in adults with moderate-to-severe atopic dermatitis inadequately controlled by conventional treatments - a randomized, double-blind, phase 2a trial. J Am Acad Dermatol. 2018;78(872e81.e6). doi:10.1016/j.jaad.2018.01.016.

52. Guttman-Yassky E, Krueger JG. Atopic dermatitis and psoriasis: two different immune diseases or one spectrum? Curr Opin Immunol. 2017;48:68e73. doi:10.1016/j.coi.2017.08.008

53. Esaki H, Brunner PM, Renert-Yuval Y, et al. Early onset pediatric atopic dermatitis is TH2 but also TH17 polarized in skin. $J$ Allergy Clin Immunol. 2016;138:1639e51. doi:10.1016/j. jaci.2016.07.013

54. Noda S, Suarez-Farinas M, Ungar B, et al. The Asian atopic dermatitis phenotype combines features of atopic dermatitis and psoriasis with increased $\mathrm{TH} 17$ polarization. $J$ Allergy Clin Immunol. 2015;136:1254e64. doi:10.1016/j.jaci.2015.04.010

55. Suarez-Farinas M, Dhingra N, Gittler J, et al. Intrinsic atopic dermatitis shows similar TH2 and higher TH17 immune activation compared with extrinsic atopic dermatitis. J Allergy Clin Immunol. 2013;132:361e70. doi:10.1016/j.jaci.2013.04.046
56. Chang SH, Reynolds JM, Pappu BP, Chen G, Martinez G, Dong C. Interleukin-17C promotes Th17 cell responses and autoimmune disease via interleukin-17 receptor E. Immunity. 2011;35:611e21. doi:10.1016/j.immuni.2011.09.010

57. Guttman-Yassky E, Krueger JG. IL-17C: A unique epithelial cytokine with potential for targeting across the spectrum of atopic dermatitis and psoriasis. J Invest Dermatol. 2018;138(7):1467-1469. doi:10.1016/j.jid.2018.02.037

58. Vandeghinste N, Klattig J, Jagerschmidt C, et al. Neutralization of IL$17 \mathrm{C}$ reduces skin inflammation in mouse models of psoriasis and atopic dermatitis. $J$ Invest Dermatol. 2018;138(7):1555-1563. doi:10.1016/j.jid.2018.01.036

59. Brembilla NC. Senra L. Boehncke W. The IL-17 family of cytokines in psoriasis: IL-17A and beyond. Front Immunol. 2018;9:1682. doi:10.3389/fimmu.2018.01682

60. Megna M, Balato A, Napolitano M, et al. Psoriatic disease treatment nowadays: unmet needs among the "jungle of biologic drugs and small molecules". Clin Rheumatol. 2018;37:1739-1741. doi:10.1007/ s10067-018-4090-6

61. Raimondo A, Balato A, Megna M, et al. Limitations of current monoclonal antibodies for plaque-type psoriasis and an outlook for the future. Expert Opin Biol Ther. 2018;18(6):605-607.

62. Talamonti M, Galluzzo M, Bianchi L, Boca AN, Costanzo A, Chimenti S. What happened after the clinical trials: long-term safety and efficacy of ustekinumab in daily clinical practice. Dermatology. 2014;229(4):324-332. doi:10.1159/000365077

63. Talamonti M, D’Adamio S, Bianchi L, Galluzzo M. The role of pharmacogenetics in chronic plaque psoriasis: update of the literature. Mol Diagn Ther. 2017;21(5):467-480. doi:10.1007/s40291-0170274-z
Clinical, Cosmetic and Investigational Dermatology

\section{Publish your work in this journal}

Clinical, Cosmetic and Investigational Dermatology is an international, peer-reviewed, open access, online journal that focuses on the latest clinical and experimental research in all aspects of skin disease and cosmetic interventions. This journal is indexed on CAS
The manuscript management system is completely online and includes a very quick and fair peer-review system, which is all easy to use. Visit http://www.dovepress.com/testimonials.php to read real quotes from published authors. 